\title{
Estrategia de unidades de salud familiar: su impacto en la tasa de embarazo en adolescentes en Paraguay
}

\author{
Domingo Santiago Ávalos, ${ }^{1}$ Felipe Recalde, ${ }^{2}$ César Cristaldo, ${ }^{3}$ Antonio \\ Cusihuaman Puma, ${ }^{4}$ Pedro López ${ }^{5}$ y Liuba Alonso Carbonel/6
}

Forma de citar

Ávalos DS, Recalde F, Cristaldo C, Cusihuaman Puma A, López P, Alonso Carbonell L. Estrategia de unidades de salud familiar: su impacto en la tasa de embarazo en adolescentes en Paraguay. Rev Panam Salud Publica. 2018;42:e59. https:/ /doi.org/10.26633/RPSP.2018.59

RESUMEN Objetivo. Determinar el impacto de la Estrategia de Unidades de Salud Familiar para la Atención Primaria de Salud en la reducción del embarazo en adolescentes en el Paraguay.

Métodos. Estudio ecológico que incluyó 18 regiones sanitarias. Se establecieron dos etapas (2000-2008 y 2008-2015). La tasa de embarazos en adolescentes se determinó a partir de los recién nacidos de madres adolescentes (<20 años) entre el total de nacidos vivos, y la cobertura poblacional de atención primaria de salud según el número de total de individuos que habitan territorios con implementación de la estrategia de atención primaria de salud y el total de habitantes del lugar del distrito por 100, para un año determinado. Se utilizó el coeficiente de correlación de Pearson.

Resultados. Durante la primera etapa, la variación porcentual de la tasa de embarazo en la adolescencia del país fue positiva en 11,5\%, y en la etapa 2 fue negativa en 14,9\%. Se encontró una relación lineal inversa entre las variables exploradas $(r=-0,654)$.

Conclusiones. Tras la implementación de la estrategia de atención primaria de salud, se ha constatado una tendencia a la disminución del embarazo en la adolescencia, lo que puede considerarse un impacto positivo a pesar de que el resultado que puede estar influido por otros factores no considerados en la investigación.

Palabras clave Atención primaria de salud; embarazo en adolescencia; Paraguay.

El embarazo en la adolescencia implica un daño físico para el cuerpo de la madre, que aún está en desarrollo, por ello estos embarazos son considerados de

\footnotetext{
Área de Educación e Investigación de la Dirección General de Atención Primaria de Salud, Ministerio de Salud Pública y Bienestar Social, Paraguay.

2 Consultor independiente.

3 Dirección General de Atención Primaria de Salud. Ministerio de Salud Pública y Bienestar Social, Paraguay.

4 Departamento de Educación, Dirección General de Atención Primaria de Salud, Ministerio de Salud Pública y Bienestar Social, Paraguay.
}

alto riesgo tanto para la madre como para el hijo (1). A nivel social, la maternidad en la adolescencia puede convertirse en un obstáculo para el acceso a

\footnotetext{
Consultor en Sistemas y Servicios de Salud Organización Panamericana de la Salud/ Organización Mundial de la Salud, Paraguay. Enviar correspondencia a Pedro López, lopezped@paho.org

6 Departamento de Ensayos Clínicos. Centro Nacional Coordinador de Ensayos Clínicos, Cuba.
}

oportunidades sociales y culturales (2), sin obviar la afectación que puede producir en el recién nacido (RN).

Según la Organización Mundial de la Salud (OMS), 16 millones de jóvenes de 15 a 19 años y aproximadamente un millón de niñas menores de 15 años dan a luz cada año. Además, tres millones de jóvenes de 15 a 19 años se someten a abortos inseguros (3). Según las Estadísticas Sanitarias Mundiales 2014, la tasa media de natalidad entre las adolescentes de 15 a 19 años es de 49 por 
cada 1000 . Las tasas de los países oscilan entre 1 y 299 nacimientos por cada 1000 adolescentes (3).

En América Latina y el Caribe (ALC), se observan menores tasas de embarazos antes de los 18 años (18\%) que las reportadas en África y Asia (28\%) y similares a la de los Estados Unidos de América (19\%); sin embargo, los porcentajes son bastante más altos en comparación con Europa y Asia Central (8\%) y los datos de Asia del Este (4\%) (3,4). A nivel global, la frecuencia de esta condición es variable y oscila entre $1,9 \%$ del total de nacimientos en los países escandinavos (desarrollados) y $26 \%$ en algunos países más pobres, con proporciones más altas cuando se analizan datos en áreas rurales (5).

En Colombia, un estudio del 2013 reporta una de las mayores tasas de embarazo adolescente de ALC, donde una de cada cinco adolescentes de entre 15 y 19 años ha estado embarazada; esto es más grave en víctimas del desplazamiento, grupo en el que $63 \%$ de las adolescentes se embarazan de su primer hijo antes de cumplir los 19 años (6). En México, se registran 64,2 partos por cada mil nacimientos en mujeres de entre 15 y 19 años. Asimismo, según Escudero Martínez, las cifras reportadas por el Instituto Nacional de Estadística y Geografía en 2010 muestran que 11682 niñas de entre 10 y 14 años dieron a luz en el país $(7,8)$. En Argentina, el porcentaje de RN de madres adolescentes respecto al total de nacimientos se ha mantenido en valores cercanos a $15 \%$ en los últimos 20 años (9).

En Paraguay, según el Fondo de Población de las Naciones Unidas (UNFPA, por sus siglas en inglés), 20\% de los embarazos corresponde a adolescentes (10). Según el Informe de Salud de las Américas de 2013, 18,3\% de los nacimientos ocurrió en madres adolescentes (11). A esto se suma que $10,2 \%$ de las adolescentes entre 15 y 19 años han tenido al menos un embarazo. En el país, la tasa de fecundidad adolescente es de 63 nacimientos por cada 1000 mujeres. Según el Sistema Nacional de Información en Salud, el porcentaje de embarazo adolescente fue de 19,9\% en el 2011 (12). Para hacer frente a la situación en el país, existen disponibles condones y anticonceptivos hormonales sin prescripción en la mayoría de las farmacias. El primero es el método preferido para la iniciación, mientras que después de la primera relación se incrementa el uso de anticonceptivos orales e inyectables. La mayoría de las intervenciones desarrolladas para adolescentes están dirigidas a las mujeres y se han enfocado en la educación orientada a las escuelas secundarias locales, con la provisión de charlas o presentaciones educacionales, a estudiantes, sus padres y sus profesores (13). Sin embargo, el Plan Nacional de Salud Sexual y Reproductiva 20142018 resalta la necesidad de apoyo técnico intersectorial y del Ministerio de Salud Pública y Bienestar Social (MSPBS) a la implementación efectiva de la educación de la sexualidad (14). La meta principal de la estrategia regional de la Organización Panamericana de la Salud (OPS) es contribuir al mejoramiento de la salud de los jóvenes mediante el desarrollo y fortalecimiento de una respuesta integrada del sector de la salud y la ejecución de programas eficaces de promoción, prevención y atención de salud de los adolescentes y los jóvenes (15).

Con base en lo anterior, se introdujeron modificaciones en la región para fortalecer la atención integral a la salud a partir de un primer nivel de atención resolutivo. Esto cambió el objetivo de la atención de la salud en ese nivel y trasladó la prioridad de la atención a la promoción de la salud y a la prevención de la enfermedad (16).

El sistema de Salud en Paraguay se caracterizó históricamente por la segmentación y la coexistencia de subsectores con distintas modalidades de financiamiento, afiliación y provisión. Además, una gran proporción de la población (cerca de $40 \%$ ) sin acceso a servicios de salud, una fuerte discriminación entre el área urbana y rural, amplios sectores de población excluidos de los servicios, falta de medicamentos, laboratorios y recursos humanos, entre otros, concentrado en un enfoque de salud muy curativo en detrimento de acciones en promoción y prevención.

Desde el año 2009, Paraguay apuesta por un Sistema de Salud basado en la atención primaria de salud (APS), con el fin de resolver las brechas existentes y garantizar el acceso y cobertura universal de salud. La nueva estrategia de APS se implementa con la creación de unidades de salud de la familia (USF), que es la estructura física y funcional en la que se desarrolla una parte importante de la estrategia de forma coordinada, integral, continuada, permanente y con base en el trabajo de un equipo de salud familiar (ESF), integrado por un médico, un enfermero, un auxiliar y entre tres y cinco agentes comunitarios. Este equipo asume la responsabilidad sanitaria y social de la atención de una comunidad definida desde los puntos de vista demográfico y geográfico, y en sus actividades han de estar contemplados tantos los aspectos de prevención de la enfermedad y promoción de la salud como la presentación de servicios asistenciales o curativos (17).

En este marco, la APS juega un papel en la prevención del embarazo en la adolescencia ya que, desde la consulta o la visita a la familia, la comunidad, o ambas, los profesionales de la salud pueden centrar su actuación en las actividades individuales, tanto en el campo de las recomendaciones y consejos como en el del cribado de población de riesgo (18).

El desarrollo de una estrategia de servicios de salud basados en la APS genera mejoras que se traducen en un incremento de las actividades preventivas y en una mejor atención (18), y se convierte en la clave para lograr un sistema de salud eficiente, que combine acciones de prevención y diagnóstico e intervención tempranos $(19,20)$ y desempeñe un papel importante en la atención de adolescentes, así como en la supervivencia y desarrollo de sus hijos (21).

Muchos adolescentes encuentran obstáculos cuando utilizan los servicios de salud y es precisamente la APS la que permite, con un enfoque integrador, superar estas barreras (19). En las estrategias exitosas para prevenir el embarazo en la adolescencia, los médicos de familia son importantes para comprometer a los adolescentes en debates confidenciales, abiertos y desprovistos de temores sobre la salud reproductiva, el comportamiento sexual responsable y el uso de contracepción (22).

A partir de todo lo comentado, se realiza esta investigación para determinar el impacto de la implementación de las USF y los ESF para la APS en Paraguay en la reducción del embarazo en adolescentes, y se toma como referencia el comportamiento de este indicador antes y después de la implementación de la estrategia.

\section{MATERIALES Y MÉTODOS}

Se realizó un estudio ecológico utilizando como fuente los datos proporcionados por la Dirección General de 
Información Estratégica-MSPBS. Se consideraron dos etapas, la etapa 1 entre el año 2000 y el 2008 y la etapa 2 entre el año 2008 y el 2015. Se estudiaron las 18 regiones sanitarias y para el estudio de correlación se incluyeron 201 municipios, con $\geq 10000$ habitantes y cuyas USF fueron habilitadas en el período de estudio.

Las poblaciones de las regiones estudiadas totalizan 6755757 habitantes y su distribución por regiones se muestra en el cuadro 1.

La tasa de embarazos adolescentes (TEA) se estableció a partir de los recién nacidos de madres adolescentes $(<20$ años) entre el total de nacidos vivos.

$$
\text { TEA }=\frac{\text { No. NVMA }}{\text { Total NV }} \times 100
$$

NVMA: nacidos vivos de madres adolescentes ( $<20$ años)

NV: nacidos vivos.

Para determinar el comportamiento de las tasas de embarazos en adolescentes antes y después de la implementación de la estrategia de APS en el Paraguay, se calcularon los porcentajes de variación a partir de la siguiente ecuación:

$$
\% \triangle T E A=\frac{(T E A A f-T E A A i)}{T E A A i} \times 100
$$

$\% \Delta$ TEA: porcentaje de variación de la TEA entre dos años de estudio de la etapa establecida (etapa $1=2000$ a 2008; etapa $2=2008$ a 2015).

TEA Ai: tasa de embarazo en adolescentes en el año inicial de la etapa establecida (etapa $1=2000$; etapa $2=2008$ ).

TEA Af: tasa de embarazo en adolescentes en el año final de la etapa establecida (etapa 1 = 2008; etapa 2 = 2015).

Para explorar la posible correlación entre la TEA y la cobertura poblacional bajo la estrategia de APS (CPAPS), se utilizó el coeficiente de correlación de Pearson (23). La variable predictora fue la CPAPS, la variable dependiente fue la TEA.

La CPAPS se define como el total de individuos que habitan territorios con implementación de la estrategia de AP entre el total de habitantes del lugar del distrito por 100 para esta investigación en particular se utilizaron datos del año 2015.

CPAPS $(\%)=\frac{\begin{array}{c}\text { No. habitantes bajo } \\ \text { estrategia APS }\end{array}}{\text { Total de habitantes }} \times 100$

La investigación respetó los postulados éticos de la declaración de Helsinki.
Se trabajó solo con información estadística proveniente de las bases de datos de fuentes reconocidas del MSPBS. Los resultados no serán empleados para otros fines fuera del marco de la investigación. No se declaran conflictos éticos.

\section{RESULTADOS}

En la figura 1 se muestra que, a nivel nacional la tasa de NV de madres adolescentes fue del 18,7 por $100 \mathrm{NV}$ en el año 2000 y de 20,8 en el 2008; se observa un aumento relativo ( $\triangle$ \% TEA $)$ de $11,5 \%(P<$ $0,001)$ en la primera etapa, hecho que experimentan la mayoría de las regiones sanitarias del país. Luego de la implementación de la estratégica de APS, se destaca que, al analizar el comportamiento entre los años 2008 y 2015, la tasa de NV de madres adolescentes disminuye en forma significativa a $17,7 \%$, con una disminución relativa de $14,9 \%(P<0,001)$.

Dichas variaciones relativas para el primer período de estudio se aprecian en la figura 2. Catorce de 18 regiones presentaron incrementos; aquellos con mayores incrementos fueron Boquerón $(71,5 \%)$ y Amambay (36,3\%). Las regiones como Misiones, Neembucú, Alto Paraguay y Asunción mostraron variaciones porcentuales negativas, es decir que disminuyó la TEA en el año final de la etapa (2008).

\section{CUADRO 1. Distribución de la población y número de unidades de salud familiar por regiones, Paraguay, 2015}

\begin{tabular}{lcc}
\hline Región & Población (habitantes) & USF \\
\hline Alto Paraguay & 16582 & 8 \\
Alto Paraná & 773303 & 77 \\
Amambay & 159263 & 21 \\
Asunción & 526408 & 33 \\
Boquerón & 60402 & 11 \\
Caaguazú & 534522 & 44 \\
Caazapá & 179576 & 49 \\
Canindeyú & 212637 & 48 \\
Central & 1985384 & 104 \\
Concepción & 236959 & 48 \\
Cordillera & 291311 & 43 \\
Guairá & 216335 & 56 \\
Itapuá & 576577 & 59 \\
Misiones & 120576 & 36 \\
Neembucú & 87750 & 25 \\
Paraguarí & 252255 & 47 \\
Presidente Hayes & 116536 & 18 \\
San Pedro & 409381 & 68 \\
\hline
\end{tabular}

USF, unidades de salud familiar.

Fuente: elaborado por los autores sobre la base de datos provistos por la Dirección General de Información Estratégica en Salud, Subsistema de Información de Estadísticas Vitales.
Luego de la instalación de las USF, la variación porcentual en cuanto a reducción de embarazo en la adolescencia fue negativa en $14,9 \%$ en todo el país (figura 3). En 16 regiones sanitarias, más el distrito capital, se obtuvieron reducciones de embarazo en adolescentes en valores porcentuales entre $12,3 \%$ y $27,2 \%$. Solo en la región Caazapá el porcentaje de variación de la TEA fue positivo $(4,4 \%)$.

En la figura 4 se observa una buena relación lineal inversa entre la CPAPS y el porcentaje de variación de la TEA antes y después de la implementación de la estrategia $(r=-0,654 ; P<0,001)$.

\section{DISCUSIÓN}

En Paraguay, existe la percepción de que la estrategia de APS impactó de manera positiva al disminuir el embarazo en adolescentes; esto se refleja en las diferencias porcentuales de las tasas antes y después de que se establecieron las USF. No obstante, no debemos obviar otros factores como el nivel de ingreso, educativo, acceso a trabajo, origen étnico y religión, entre otros que estarían relacionados y mueven a estudios posteriores.

El fenómeno al que nos enfrentamos resulta complejo, ya que existen muchos determinantes que pueden influir en él, por ello los autores resaltan que, a pesar 
FIGURA 1. Tasa de embarazos en adolescentes según la región y en el país; Paraguay, años 2000, 2008 y 2015

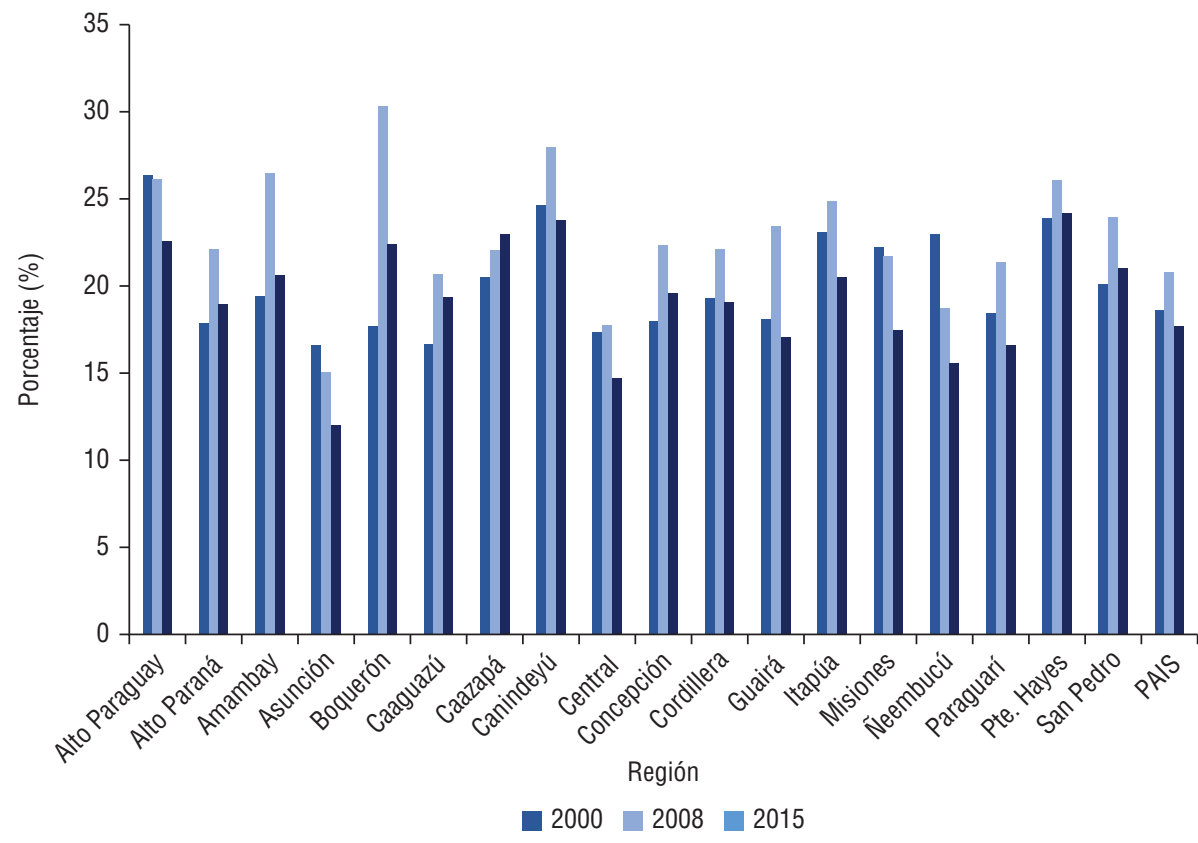

Fuente: elaborada por los autores a partir de la base de datos proporcionada por la Dirección General de Información Estratégica en Salud; Subsistema de Información de Estadísticas Vitales.

FIGURA 2. Variación porcentual de la tasa de embarazos en adolescentes, en el nivel país y departamental, previa implementación de la Estrategia de unidades de salud familiar para la atención primaria de salud, 2000-2007



Fuente: elaborado por los autores a partir de la base de datos proporcionada por la Dirección General de Información Estratégica en Salud; Subsistema de Información de Estadísticas Vitales. de que la reducción del embarazo en la adolescencia es evidente luego de la implementación de las USF, resulta inadecuado suponer que esos resultados se deben exclusivamente a esta intervención. En este sentido, deben considerarse otros factores antes mencionados, que pueden contribuir a la reducción del embarazo adolescente y no fueron incluidos en el presente trabajo; no obstante, el hallazgo de una buena correlación confirma de manera indirecta la pertinencia de la variable predictora seleccionada.

Las USF constituyen una puerta de entrada al Sistema Nacional de Salud, reduce la exclusión social y aumenta la cobertura. Además, permite actuar sobre las causas sociales que generan las enfermedades, desde los territorios donde viven y se relacionan las personas (24).

El modelo propuesto por la OMS en el 2008 (25) asume que las condiciones sociales en que las personas viven y trabajan impactan sobre la salud y son las que explican la mayor parte de las inequidades sanitarias; por ello se debe actuar sobre estas condiciones para mejorar la situación global y combatir inequidades (26).

Si bien no es el único elemento a considerar, los servicios de salud contribuyen a atender los problemas de salud y los comportamientos relacionados con esta, que son frecuentes en la adolescencia. Ya se han cosechado algunos éxitos. Por ejemplo, el programa nacional de salud de los adolescentes de El Salvador ha permitido mejorar la calidad y cobertura de los servicios de atención primaria, y la tasa de fecundidad de las adolescentes ha disminuido (27).

Las actividades que se realizan en APS con los adolescentes permiten una intervención promocional educativa en relación a la sexualidad responsable, la planificación familiar y la maternidad y paternidad consciente, para favorecer la salud sexual y desarrollar un sentimiento hacia la prevención (28).

No se encontró, en la bibliografía revisada para este trabajo, literatura referente al impacto de la APS en el control del embarazo en adolescentes; sin embargo, las evaluaciones realizadas en países desarrollados para medir los impactos de las iniciativas de renovación de la APS: Canadá, el Reino Unido y Nueva Zelanda demuestran que su fortalecimiento puede incrementar el acceso y satisfacer las necesidades de salud a través de servicios integrales e integrados (29). En general, 
FIGURA 3. Variación porcentual de la tasa de embarazos en adolescentes, en el nivel país y departamental luego de implementada la Estrategia de unidades de salud familiar para atención primaria de salud, Paraguay, 2008-2015

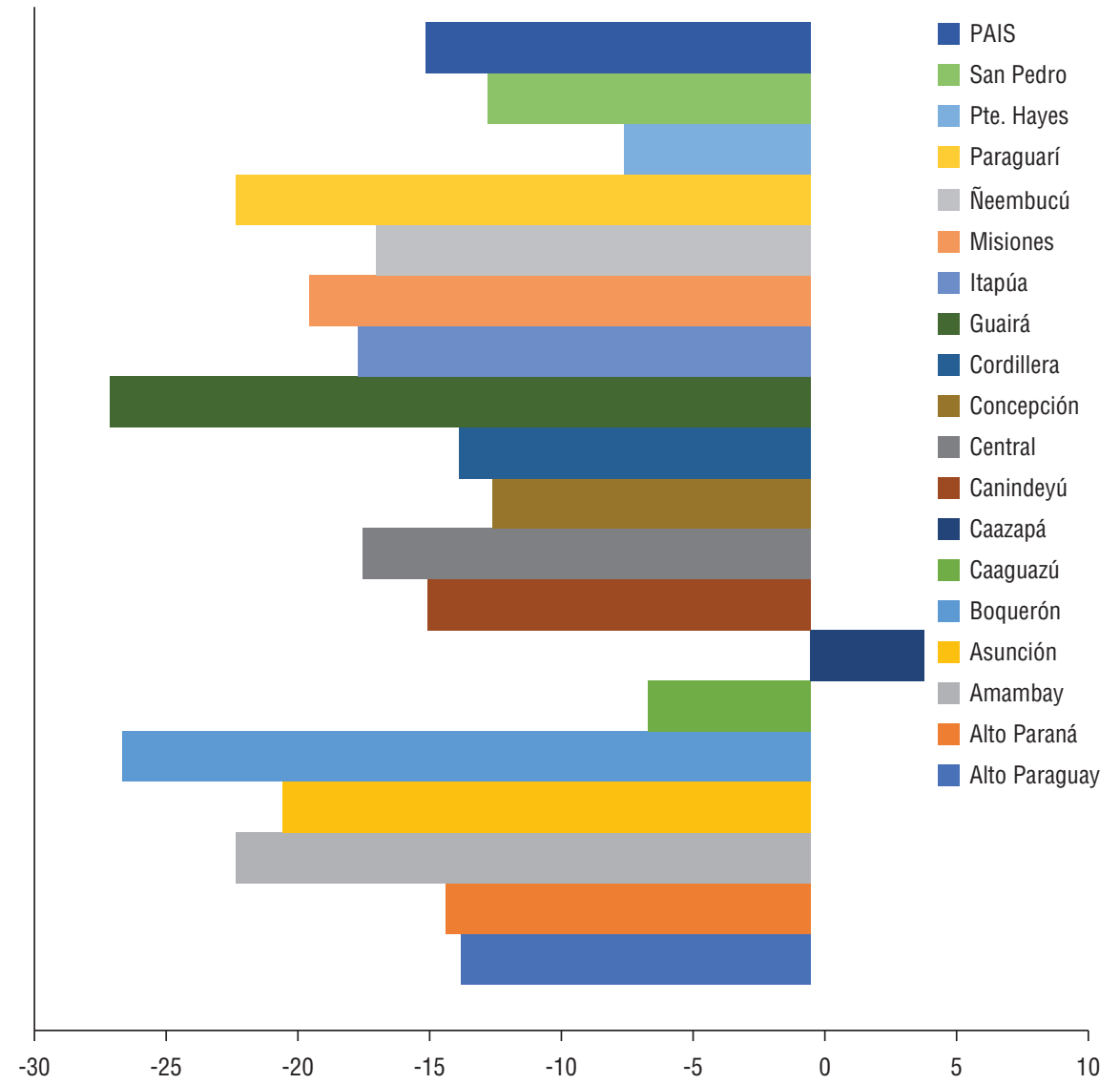

Fuente: elaborado por los autores a partir de la base de datos proporcionada por la Dirección General de Información Estratégica en Salud; Subsistema de Información de Estadísticas Vitales.

FIGURA 4. Correlación entre la cobertura poblacional por unidad de salud familiar y el porcentaje de embarazo en adolescentes en Paraguay

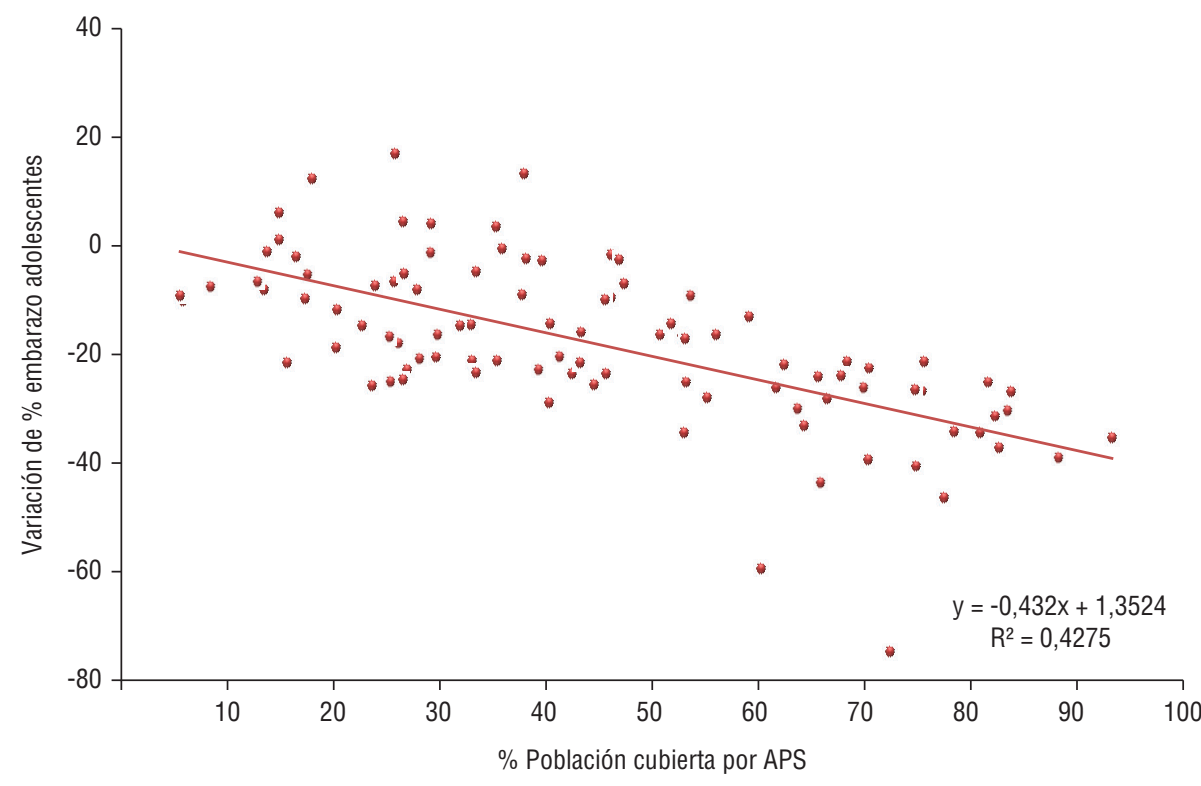

Fuente: elaborado por los autores a partir de la base de datos proporcionada por la Dirección General de Información Estratégica en Salud; Subsistema de Información de Estadísticas Vitales. los sistemas basados en la APS son capaces de mejorar la equidad porque su enfoque es menos costoso para los individuos y más rentable para la sociedad en comparación con los sistemas orientados hacia la atención especializada. Por consiguiente, puede ayudar a liberar recursos para atender las necesidades de salud de los individuos que se encuentran en peor situación $(30,31)$.

En ALC, las experiencias de APS han sido determinantes en el desarrollo de servicios de salud: en Argentina registran experiencias basadas en APS como estrategia de atención dentro del sistema de salud como el caso de la ciudad de Rosario, así como experiencias de mejoramiento a través de programas que se incorporan al sistema de salud (32). Se reconoce el caso de Brasil y su programa de salud familiar (33), considerado hoy una de las experiencias más destacables en la región.

Las limitaciones de esta investigación son las propias de los estudios ecológicos, que utilizan como unidad de análisis a ciudades o departamentos; por lo tanto, podría suceder que las adolescentes que recibieron asesoramiento por los programas en prevención de embarazos no sean las mismas que están experimentando dicha prevención. También podría ocurrir que determinantes sociales formen parte de las variables predictoras de un posible control de embarazo en este grupo etario, lo que impide establecer una correlación exacta entre las variables consideradas. Por ello, hacen falta más investigaciones, con datos más exhaustivos que apunten a determinar la magnitud y la velocidad de aparición de este evento.

Podemos concluir que, tras la implementación de las USF, se ha constatado una tendencia a la disminución del embarazo en adolescentes. Esto permite evaluar como positivo el impacto de la estrategia sobre este indicador, sin dejar de considerar los diversos aspectos que pueden influir en la pobre correlación encontrada, por lo que se requieren más estudios para confirmar dicha tendencia.

Conflictos de interés. Ninguno declarado por los autores.

Declaración. Las opiniones expresadas en este manuscrito son responsabilidad de los autores y no reflejan necesariamente los criterios ni la política de la RPSP/PJPH o de la OPS. 


\section{REFERENCIAS}

1. Manrique R, Rivero A, Ortunio M, Rivas M, Cardozo R, Guevara H. Parto pretérmino en adolescentes. Rev Obstet Ginecol Venez [serie en Internet]. 2008;68(3). Disponible en: http://www.scielo.org.ve/ scielo.php?script $=$ sci_arttext\&pid $=$ S0048-77322008000300002\&lng=es Acceso el 12 de julio de 2017.

2. Estadísticas a Propósito del Día Internacional de los Pueblos Indígenas de México. Datos Nacionales, 2015. Disponible en: http://www.inegi.org.mx/saladeprensa/ aproposito/2015/juventud0.pdf Acceso el 30 de julio de 2017.

3. Organización Mundial de la Salud (OMS). El embarazo en la adolescencia. Nota descriptiva No. 364, 2014. Disponible en: http:// www.who.int/mediacentre/factsheets / fs364/es/ Acceso el 17 de julio de 2017.

4. Barcelata BE, Farías S, Rodríguez R. Embarazo adolescente: una mirada al funcionamiento familiar en un contexto urbano-marginal. Revista Eureka [serie en Internet]. 2014;11(2). Disponible en: http://psicoeureka.com.py/ sites/default/files/articulos/eureka-11-2-8. pdf Acceso el 30 de julio de 2017.

5. Instituto Mexicano para la competitividad (IMCO). A.C. Competitividad Internacional, 2011 Consejo directivo IMCO. Disponible en: http://imco.org.mx/images/pdf/Indicede-Competitividad-Internacional-2011.pdf Acceso el 20 de julio de 2017.

6. Sánchez YA, Mendoza LA, Grisales MB, Ceballos LY, Bustamante JC, Muriel E, et al. Características poblacionales y factores asociados a embarazo en mujeres adolescentes de Tuluá, Colombia. Rev Chil Obstet Ginecol. [serie en Internet]. 2013;78(4). Disponible en: http://www.scielo.cl/pdf/rchog/v78n4/ art05.pdf Acceso el 25 de julio de 2017.

7. Escudero M. Estrategia Nacional de Prevención del embarazo en adolescente. La propuesta y sus retos. Rev Educ \& Cultura. [en Internet]. 2015;92. Disponible en: http:/ / www.educacionyculturaaz.com / 092/ 092-AZABRIL2015.pdf Acceso el 28 de noviembre de 2017.

8. Velásquez CG. Mujeres mexicanas. Rev Mex Reprod. 2011;3:139-41.

9. Ministerio de Salud de la Nación Argentina y Organización Panamericana de la Salud Indicadores Básicos. Argentina, 2012. Disponible en http://www.msal.gov.ar/ images/stories/pdf/indicadores-basicos-2012.pdf Acceso el 20 de julio de 2017.

10. Fondo de Población de las Naciones Unidas (UNFPA). Embarazos adolescentes, rostro de la desigualdad. Revista JOPARÉ. [en Internet]. 2013;53. Disponible en: http:// www.unfpa.org.py/download/jopare53. pdf Acceso el 23 de julio de 2017.

11. Organización Panamericana de la Salud/ Organización Mundial de la Salud (OPS/ OMS). Salud en las Américas. Informe de país: Paraguay, 2017. Disponible en: http:/ / www.paho.org/salud-en-las-americas-2017/?page_t_es=informes $\% 20 \mathrm{de} \% 20$ pais/paraguay\&lang=es Acceso el 20 de octubre de 2017.

12. Ministerio de Salud Pública y Bienestar Social de Paraguay. Dirección General de Información Estratégica en Salud. Sistema
Nacional de Información en Salud. 2011. Disponible en: http:/ / www.mspbs.gov.py/ indicadoresdesalud/indicadores-de-morbilidad/ Acceso el 24 de setiembre de 2017.

13. Centro Paraguayo de Estudios de Población. Salud Sexual y Reproductiva en Adolescentes y Jóvenes, Resultados en base al análisis de la Encuesta Nacional de Salud Sexual y Reproductiva 2008. 2011. Disponible en: http://www.cepep.org. py/archivos/InformeADOLESCENTES. pdf Acceso el 2 de diciembre de 2017.

14. Ministerio de Salud Pública y Bienestar Social de Paraguay. Plan Nacional de Salud Sexual y Reproductiva. Paraguay 2014-2018. Disponible en: http://www. cepep.org.py/archivos/PNSSR2014.pdf Acceso el 24 de noviembre de 2017.

15. Organización Panamericana de la Salud (OPS). Estrategia y plan de acción regional sobre los Adolescentes y Jóvenes 2010 2018. Disponible en: http://new.paho. org/hq/dmdocuments/2011/Estrategiay-Plan-de-Accion-Regional-sobre-losAdolescentes-y-Jovenes.pdf Acceso el 20 de julio de 2017.

16. Villar M. Factores determinantes de la salud: importancia de la prevención. Acta Med Peruana [en Internet]. 2011;28(4). Disponible en: <http://www.scielo.org. pe/scielo.php?script=sci_arttext\&pid$=$ S1728-59172011000400011\&lng $=$ es\&nr$\mathrm{m}=$ iso $>$. ISSN 1728-5917 Acceso el 28 de julio de 2017.

17. Ministerio de Salud Pública y Bienestar Social de Paraguay (MSPBS). Manual de funciones de las unidades de salud de la familia. Asunción: MSPBS; 2006.

18. Colomer J. Prevención del embarazo en la adolescencia. Rev Pediatr Aten Primaria [en Internet]. 2013;15(59). Disponible en: http:/ / scielo.isciii.es/scielo.php?script=sci_arttext\&pid=S1139-76322013000400016\&ln$\mathrm{g}=$ es Acceso el 28 de julio de 2017.

19. Barbaro MC, Lettiere A, Spanó Nakano AM. Asistencia prenatal a la adolescente y los atributos de la Atención Primaria a la Salud. Rev Latino-Am Enfermagem [en Internet]. 2014;22(1). Disponible en: http:/ / www.scielo.br/pdf/rlae/v22n1/ es_0104-1169-rlae-22-01-00108.pdf Acceso el 24 de junio de 2017.

20. Franco-Giraldo A. El rol de los profesionales de la salud en la atención primaria en salud (APS). Rev Fac Nac Salud Pública [en Internet]. 2015;33(3). Disponible en: http:/ / www.scielo.org.co/pdf/rfnsp/v33n3/ v33n3a11.pdf Acceso el 24 de junio de 2017.

21. López Y. Embarazo en la adolescencia y su repercusión biopsicosocial sobre el organismo de la madre y de su futuro hijo. Revista Cubana de Enfermería [en Internet]. 2011;27(4). Disponible en: http:/ / scielo.sld.cu / pdf/enf/v27n4/ enf11411.pdf Acceso el 20 de julio de 2017.

22. Hurtado F, Donat F, Colomer J, Pla E, Sánchez A, Sarabia S, et al. Promoción, prevención, detección y actuación ante embarazos no deseados e infecciones de transmisión sexual en adolescencia desde atención primaria. C Med Psicosom [en Internet]. 2014;111. Disponible en: https:/ / dialnet.unirioja.es/servlet/articulo?codigo=4906940 f Acceso el 2 de julio de 2017 .

23. Johnson R. Estadística elemental. 3ra ed. Mexico DF: Ed. Math Learning; 2003.

24. Guillen MC. Paraguay. Sistemas de salud en Sudamérica: desafíos hacia la integralidad y equidad. Paraguay: Ministerio de Salud Pública y Bienestar Social; 2011. Disponible en: http://www.mspbs.gov. py / planificacion / w p-content / uploads/2012/07/SSS-PARAGUAY-2011. pdf Acceso el 21 de julio de 2017.

25. Organización Panamericana de la Salud (OMS). Comisión sobre Determinantes Sociales de la Salud. "Subsanar las desigualdades en una generación". Informe final. Ginebra: OMS; 2008.

26. Frenz P. Desafíos en Salud Pública de la Reforma: equidad y determinantes sociales de la salud. Santiago de Chile: Ministerio de Salud; 2005.

27. Organización Mundial de la Salud (OMS=. Salud para los adolescentes del mundo: una segunda oportunidad en la segunda década. Ginebra: OMS; 2014. Disponible en. http:/ /apps.who.int/adolescent/seconddecade/files/WHO_FWC_MCA_14.05_ spa.pdf Acceso el 31 de julio de 2017.

28. Quintero PP, Castillo N, Roba BC, Padrón O, Hernández ME. Estrategia de intervención educativa para prevenir el embarazo en la adolescencia. Rev Ciencias Médicas [en Internet]. 2012;16(1). Disponible en: http://scielo.sld.cu/scielo.php?script=sci_ arttext\&pid=S1561-31942012000100015\&ln$\mathrm{g}=\mathrm{es}$ Acceso el 17 de enero de 2017.

29. Ministerio de Salud Pública y Bienestar Social de Paraguay. Dirección de Investigación y Estudios Estratégicos. Rol de la APS en el abordaje integrado para el manejo de las enfermedades crónicas no transmisibles: opciones de políticas de salud en el contexto del sistema de salud del Paraguay. Asunción: OPS, 2011.

30. Starfield B, Shi L, Macinko J. Contribution of primary care to health systems and health. Milbank Q. 2005;83:457-50.

31. Macinko J, Guanais F, Marinho F. An evaluation of the impact of the family health program on infant mortality in Brazil, 1990-2002. Journal of Epidemiology and Community Health. 2006;60(1):13-19.

32. Marin G, Silberman M, Etchegoyen G. Programa de Atención Personalizada y Nominal de la Salud-PANDELAS. Buenos Aires, Argentina, 2006. Rev Salud Publica. 2008;10(2):203-14.

33. De Almeida P, Giovannella L. Avaliação em atenção Básica a Saúde no Brasil: mapeamento e análise das pesquisas realizadas e/ou financiadas pelo Ministério da Saúde entre os anos 2000-2006. Cad. Saúde Publica. 2008;24(8):1727-42.

Manuscrito recibido el 4 de agosto de 2017. Aceptado para publicación, tras revisión, el 22 de diciembre de 2017. 
ABSTRACT Objective. To determine the impact of the Family Health Units Strategy for Primary Health Care in reducing adolescent pregnancy in Paraguay.

Methods. Ecological study that included 18 health regions. Two stages were established (2000-2008 and 2008-2015). The adolescent pregnancy rate was established based

\section{Impact of the Family Health Units Strategy for Primary Health Care on adolescent pregnancy rate in Paraguay} on the newborns of adolescent mothers ( $<20$ years) and the total of live births, and the population coverage of primary health care based on the total number of individuals living in areas where the strategy of primary health care was implemented and the total number of inhabitants of the district, for a given year. The Pearson correlation coefficient was used.

Results. During the first stage, the percentage variation of the adolescent pregnancy rate in the country was positive at $11.5 \%$, and in stage 2 it was negative at $14.9 \%$. An inverse linear relationship was found between the variables explored $(r=-0.654)$.

Conclusions. After the implementation of the primary health care strategy, there has been a trend towards a decrease in adolescent pregnancy, which can be considered a positive impact even though the result may be influenced by other factors not considered in the study.

Keywords Primary health care; pregnancy in adolescence; Paraguay.
RESUMO

Impacto da Estratégia das Unidades de Saúde da Família para Atenção Primária a Saúde na taxa de gravidez na adolescência no Paraguai

Palavras-chave
Objetivo. Determinar o impacto da Estratégia das Unidades de Saúde da Família para Atenção Primária a Saúde na redução da gravidez na adolescência no Paraguai. Métodos. Estudo ecológico que incluiu 18 regiões de saúde. Foram estabelecidas duas etapas (2000-2008 e 2008-2015). A taxa de gravidez na adolescência foi estabelecida a partir dos recém-nascidos de mães adolescentes ( $<20$ anos) entre o total de nascidos vivos, e a cobertura populacional de atenção primária a saúde a partir do número total de indivíduos que vivem em territórios com implementação da estratégia de atenção primária a saúde e o número total de habitantes do distrito por 100, para um ano determinado. Foi utilizado o coeficiente de correlação de Pearson.

Resultados. Durante o primeiro estágio, a variação percentual da taxa de gravidez na adolescência no país foi positiva em $11,5 \%$, e no segundo estágio foi negativa em $14,9 \%$. Foi encontrada uma relação linear inversa entre as variáveis exploradas $(\mathrm{r}=-0.654)$.

Conclusões. Após a implementação da estratégia de atenção primária a saúde houve uma tendência para uma diminuição da gravidez na adolescência que pode ser considerada um impacto positivo, apesar do fato de que o resultado pode ser influenciado por outros fatores não considerados no estudo.

Atenção primária à saúde; gravidez na adolescência; Paraguai. 